\title{
Differential susceptibility of gastric cancer cells to TRAIL-induced apoptosis
}

\author{
NAK-MI SONG ${ }^{1}$, SUYEON JUN ${ }^{1}$, DAE YOUNG ZANG ${ }^{2}$, SUNG GYUN KIM ${ }^{2,3}$, \\ HYE-RIM PARK $^{4}$ and DONGCHUL KANG ${ }^{1}$ \\ ${ }^{1}$ Ilsong Institute of Life Science, Hallym University; ${ }^{2}$ Department of Internal Medicine, ${ }^{3}$ Kidney Research Institute, \\ ${ }^{4}$ Department of Pathology, Hallym University Medical School and Hallym University \\ Sacred Heart Hospital, Anyang, Kyonggi-do 431-060, Republic of Korea
}

Received September 17, 2012; Accepted November 23, 2012

DOI: $10.3892 /$ or.2012.2183

\begin{abstract}
Understanding the molecular basis of the differential sensitivity of cancer cells to tumor necrosis factor-related apoptosis-inducing ligand (TRAIL)-mediated apoptosis is required to predict therapeutic outcomes and to improve the effectiveness of TRAIL-based therapy. This study aimed to compare the responsiveness of gastric cancer cells to TRAIL treatment and to investigate the molecular basis of the differential TRAIL sensitivity of four gastric cancer cell lines. The TRAIL sensitivity of the four cell lines was ranked in the following order: SNU-16 SNU-620 > SNU-5 > SNU-1. The level of Annexin V binding and the activation profile of caspase- $3,-8$ and -9 corroborated the differential TRAIL susceptibility of the cell lines. To determine the molecular basis of the differential sensitivity to TRAIL, we examined the expression of signaling components involved in TRAIL-mediated apoptosis. The mRNA level and surface expression of death receptor 4 (DR4) were significantly decreased in the SNU-1 cells compared to the other cell lines. Bid cleavage and X-linked inhibitor of apoptosis (XIAP) degradation were significantly increased in the SNU-16 and SNU-620 cells compared to the SNU-5 and SNU-1 cells, although Bid and XIAP were expressed at similar levels across the four cell lines. The expression and degradation of FLICE-inhibitory protein (FLIP) upon TRAIL treatment was independent of TRAIL sensitivity. In conclusion, the differential susceptibility of the four gastric cancer cells to TRAIL may be ascribed to the differential expression of DR4 and the proper augmentation of the death signal by the truncation of Bid and degradation of XIAP.
\end{abstract}

\section{Introduction}

Tumor necrosis factor-related apoptosis-inducing ligand (TRAIL, also termed APO-2L) is a pro-apoptotic cytokine

Correspondence to: Dr Dongchul Kang, Ilsong Institute of Life Science, Hallym University, 1605-4 Gwanyang-dong, Dongan-gu, Anyang, Kyonggi-do 431-060, Republic of Korea

E-mail: dckang@hallym.ac.kr

Key words: TRAIL, gastric cancer, apoptosis that belongs to the tumor necrosis factor superfamily (1). A TRAIL homotrimer interacts with a homotrimeric TRAIL-R1 [death receptor (DR)4] or TRAIL-R2 (DR5), initiating extrinsic and indirectly intrinsic pathways of apoptosis and nuclear facgtor- $\kappa \mathrm{B}(\mathrm{NF}-\kappa \mathrm{B})$ activation (2). TRAIL also binds to two other membrane receptors, TRAIL-R3 [decoy receptor (DcR)1] and TRAIL-R4 (DcR2) that do not contain functional death domains and function as DcRs (2). Another TRAIL receptor, osteoprotegerin (OPG), acts as a soluble DcR for TRAIL (3). TRAIL is known to play critical roles in immune surveillance and defense mechanisms against cancer cells, as well as in normal hematopoiesis $(4,5)$.

TRAIL is known to preferentially induce apoptosis in cancer cells, with little to no toxicity to normal cells, which has prompted research into its therapeutic application (6). Although TRAIL manifests fewer side-effects than conventional chemotherapeutic reagents, there are nevertheless considerable obstacles to the clinical application of TRAIL. Since the majority of injected TRAIL is rapidly cleared, a large dose of TRAIL may be required in clinical situations (7). Additionally, sensitivity to TRAIL-mediated apoptosis is widely divergent depending on the type of cancer $(8,9)$.

Cancer cells equipped with any type of anti-apoptotic mechanisms can potentially evade TRAIL-mediated apoptosis. The silencing of or loss-of-function mutations in DR4 and DR5 via genetic and epigenetic changes in cancer cells can render the cancer cells resistant to TRAIL (10-13). The increased expression of DcRs is also involved in the TRAIL resistance of cancer cells (14). In addition, cancer cells that overexpress anti-apoptotic molecules, including cellular FLICE-inhibitory protein (c-FLIP), X-linked inhibitor of apoptosis (XIAP) and Bcl-2, are resistant to TRAIL-mediated apoptosis (15-17). Since TRAIL-mediated apoptosis can be regulated by the expression status of various anti- and pro-apoptotic molecules, the focus of research on the therapeutic application of TRAIL has shifted toward the discovery of indicators and enhancers of the TRAIL sensitivity of cancer cells $(8,18,19)$.

Gastric cancer was estimated to be the fourth most common malignancy and the second leading cause of cancer-related mortality worldwide in 2008 (20). The incidence of gastric cancer is reported to be particularly high in East Asian countries, including Japan, China and Korea. Gastric cancer is treated by 
the surgical resection of the operable tumor, accompanied by localized radiotherapy and chemotherapy with conventional chemotherapeutics (21). However, the non-specific toxicity of the drugs necessitates the development of novel therapeutic measures to treat the disease, which has prompted a search for novel therapeutics (22).

TRAIL has been tested as a candidate drug for the treatment of gastric cancer (23). Although TRAIL induces the apoptosis of many gastric cancer cells, considerable resistance has also been reported in a few gastric cancer cells $(16,24)$. To optimize TRAIL utility as an effective therapeutic strategy for gastric cancer, it is necessary to identify critical indicators of TRAIL treatment outcomes and potential enhancers of TRAIL efficacy $(11,23,25,26)$. In the present study, we investigated the response of various gastric cancer cell lines to TRAIL, the mechanism of TRAIL-mediated apoptosis and the molecular basis of the differential sensitivity to TRAIL. Four gastric cancer cells grown in suspension manifested differential susceptibility to TRAIL treatment. The expression of signaling components involved in TRAIL-mediated apoptosis, including TRAIL receptors, caspases and apoptosis-modulating proteins, was examined to investigate the mechanism of TRAIL-mediated apoptosis and the molecular basis of the divergent responses to TRAIL treatment.

\section{Materials and methods}

Cell culture. The gastric cancer cell lines, SNU-1, SNU-5, SNU-16 and SNU-620, were cultured in RPMI-1640 (HyClone, South Logan, UT, USA) supplemented with $10 \%$ fetal bovine serum (HyClone), $100 \mathrm{U} / \mathrm{ml}$ penicillin and $100 \mu \mathrm{g} / \mathrm{ml}$ streptomycin (HyClone) at $37^{\circ} \mathrm{C}$ in a humidified $5 \% \mathrm{CO}_{2}$ incubator. They were grown in suspension and subcultured twice a week by splitting 1:10 after collection by centrifugation.

MTT assay. Methylthiazolyldiphenyl-tetrazolium bromide (MTT; Sigma-Aldrich, St. Louis, MO, USA) assay was carried out as previously described in the study by Huynh et al (27), with some modifications for cell suspension. A total of 2,000 cells/ well plated in a 96-well plate on the previous day were treated with either human TRAIL APO-II Ligand (PeproTech Inc., Rocky Hill, NJ, USA) or the vehicle, as specified in the figure legends. At 24, 48 or $72 \mathrm{~h}$ after treatment, $20 \mu \mathrm{l} /$ well MTT solution $(2.5 \mathrm{mg} / \mathrm{ml} \mathrm{PBS})$ were added to the cells and incubated for $3 \mathrm{~h}$. Solubilizer ( $80 \mu \mathrm{l} /$ well, $10 \%$ SDS with $0.01 \mathrm{~N} \mathrm{HCl}$ ) was added, and the plate was incubated at $37^{\circ} \mathrm{C}$ overnight to dissolve the MTT formazan. The absorbance at $570 \mathrm{~nm}$, with reference absorbance at $650 \mathrm{~nm}$, was measured with a Multiskan GO spectrophotometer (Thermo Scientific, Rockland, IL, USA). The percentage viability was calculated by [(absorbance of experimental well)/(absorbance of control well)] x100\%. The $\mathrm{IC}_{50}$ was obtained by plotting log[percentage viability] against TRAIL concentration and determining the TRAIL concentration at $50 \%$ viability.

Flow cytometric analysis. Annexin V binding and the expression of TRAIL receptors on the cell surface were analyzed by flow cytometry. For Annexin V binding analysis, cells seeded at $1.2 \times 10^{5} /$ well in 6 -well plates on the previous day were treated with $50 \mathrm{ng} / \mathrm{ml}$ TRAIL for $16 \mathrm{~h}$. The cells were collected by centrifugation (520 x g, 2 min) and were incubated in PBS containing $2 \mathrm{mM}$ EDTA and $0.5 \%$ FBS for $2 \mathrm{~min}$. The cells were washed once with Annexin $\mathrm{V}$ binding buffer and then incubated in buffer containing FITC-conjugated Annexin V (100 $\mu \mathrm{l}, 0.05 \mu \mathrm{g} / \mathrm{ml}$; Annexin V-FITC detection kit, eBioscience, San Diego, CA, USA) and propidium iodide (PI, $5 \mu \mathrm{g} / \mathrm{ml}$ ) at room temperature in the dark for $30 \mathrm{~min}$. Annexin $\mathrm{V}$ binding and PI infiltration were measured by flow cytometry using a FACSCalibur ${ }^{\mathrm{TM}}$ (BD Biosciences, Sparks, MD, USA), and the data were analyzed with CellQuest Pro $^{\mathrm{TM}}$ software (BD Biosciences). To detect cell surface receptor expression, cells, plated as described above, were allowed to grow for $48 \mathrm{~h}$. The pelleted cells ( $520 \mathrm{x} \mathrm{g,} 2 \mathrm{~min}$ ) were dispersed by incubation in PBS containing $2 \mathrm{mM}$ EDTA and $0.5 \%$ FBS for $2 \mathrm{~min}$, and the cells were then incubated in $100 \mu \mathrm{l}$ PBS containing phycoerythrin (PE)-conjugated $\alpha$-TRAIL, $\alpha$-DcR1 (CD263), $\alpha$-DR4 (CD261), $\alpha$-DR5 (all from eBioscience) or $\alpha$-DcR2 antibody (B-R27, Santa Cruz Biotechnology) for $30 \mathrm{~min}$ in the dark at room temperature. A PE-conjugated mouse $\mathrm{IgG}$ isotype control (eBioscience) was used as the negative control. The fluorescence signal from the bound antibodies was measured by flow cytometry, as described above.

RNA isolation and RT-PCR. The total RNA was isolated, and an aliquot $(5 \mu \mathrm{g})$ was reverse-transcribed, as previously described (27). A total of $2 \mu \mathrm{l}$ of each RT reaction mixture was then employed for real-time PCR using the SYBR ${ }^{\circledR}-$ Green PCR kit (Qiagen, Hilden, Germany) with LightCycler 2.0 (Roche, Basel, Switzerland) as directed by the manufacturer. The primers used in the experiments were as follows: TRAIL (GGAACCCAAGGTGGGTAGAT/TCTCA

CCACACTGCAACCTC), DR4 (CTGAGCAACGCAGACT CGCTGTCCAC/TCCAAGGACACGGCAGAGCCTGTGC CAT), DR5 (GCCTCATGGACAATGAGATAAAGGTG GCT/CCAAATCTCAAAGTACGCACAAACGG), DcR1 (GAAGAATTTGGTGCCAATGCCACTG/CTCTTGGACTT GGCTGGGAGATGTG), DcR2 (GGCTGCTGGTTCCAGT GAATGACGCT/GTTTCTTCCAGGCTGCTTCCCTTTG TAG) and GAPDH (TGATGACATCAAGAAGGTGG/TCCTT GGAGGCCATGT GGGCCAT). The PCR reactions consisted of one cycle of $95^{\circ} \mathrm{C}$ for $10 \mathrm{~min}$, followed by 45 cycles of $95^{\circ} \mathrm{C}$ for $10 \mathrm{sec}, 62^{\circ} \mathrm{C}$ for $5 \mathrm{sec}$ and $72^{\circ} \mathrm{C}$ for $20 \mathrm{sec}$. The input cDNA levels were monitored by measuring GAPDH in a parallel reaction. The specific mRNA levels were calculated by $(1 / 2)^{\Delta \Delta C}{ }_{T}$. $\Delta \Delta \mathrm{C}_{\mathrm{T}}$ is defined as $\mathrm{C}_{\mathrm{T}}$ of targets- $\mathrm{C}_{\mathrm{T}}$ of GAPDH.

Protein extraction and western blot analysis. Total cell lysates were prepared and analyzed by western blot analysis, as previously described (27). The antibodies used in the experiments were $\alpha$-caspase-9, $\alpha$-caspase- $3, \alpha$-XIAP (Cell Signaling Technology, Danvers, MA, USA), $\alpha$-BID, $\alpha$-caspase- 8 (Chemicon, Temecula, CA, USA), $\alpha$-DR5, $\alpha$-FLIP (eBioscience), $\alpha$-DR4 (Novus Biologicals, Littleton, CO, USA) and $\alpha-\beta$-actin (Bethyl Laboratories Inc., Montgomery, TX, USA).

\section{Results}

Response of gastric cancer cells to TRAIL. The responsiveness of the four gastric cancer cell lines, SNU-1, SNU-5, SNU-16 and SNU-620, to TRAIL was determined by MTT assay. TRAIL 
induced cell death in a concentration-dependent manner in all four cell lines. However, the $\mathrm{IC}_{50}$ of TRAIL at each time-point demonstrated the differential TRAIL sensitivity of the cell lines (Fig. 1A). The $\mathrm{IC}_{50}$ values at $48 \mathrm{~h}$ were $12.7 \mathrm{ng} / \mathrm{ml}$ for SNU-16, $14.0 \mathrm{ng} / \mathrm{ml}$ for SNU-620, $30.3 \mathrm{ng} / \mathrm{ml}$ for SNU-5 and $78.7 \mathrm{ng} / \mathrm{ml}$ for SNU-1. In general, the $\mathrm{IC}_{50}$ values of the SNU-16 and SNU-620 cells were 4- to 6-fold lower than those of the SNU-1 cells at each measured time-point. The TRAIL-mediated apoptosis of the gastric cancer cells was confirmed by flow cytometric analysis of the Annexin V-bound cells. In the SNU-16 and SNU-620 cells, TRAIL significantly increased the number of both Annexin Vand PI-positive cells, whereas there was no significant increase in these populations in the SNU-1 cells (Fig. 1B). The fraction of Annexin V-and/or PI-positive cells was displayed in the following order: SNU-16 $\approx$ SNU-620 > SNU-5 >> SNU-1. Both the MTT assay and flow cytometry analysis results indicated that the SNU-16 and SNU-620 cells were highly sensitive to TRAILmediated cell death, whereas the SNU-1 cells were relatively resistant and the SNU-5 cells showed intermediate sensitivity to TRAIL.

The activation of caspase- $3,-8$ and -9 upon TRAIL treatment was measured by western blot analysis (Fig. 1C). The expression levels of procaspase-3, -8 and -9 were comparable between the gastric cancer cells. Although TRAIL treatment increased the amount of active caspase- 8 fragments in all four cell lines, the quantity of the fragments in the control and TRAIL-treated cells was much higher in the TRAIL-sensitive

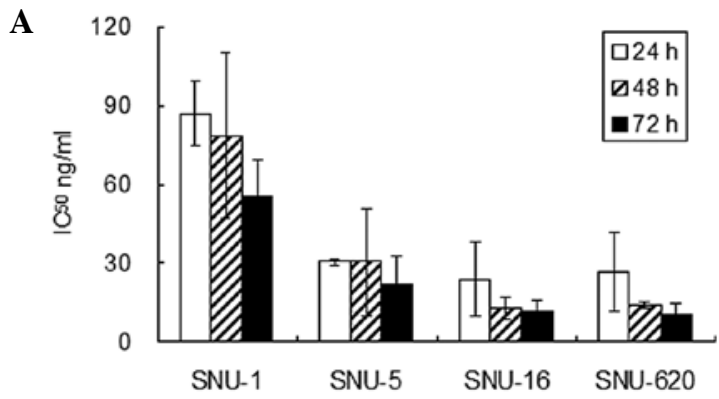

B
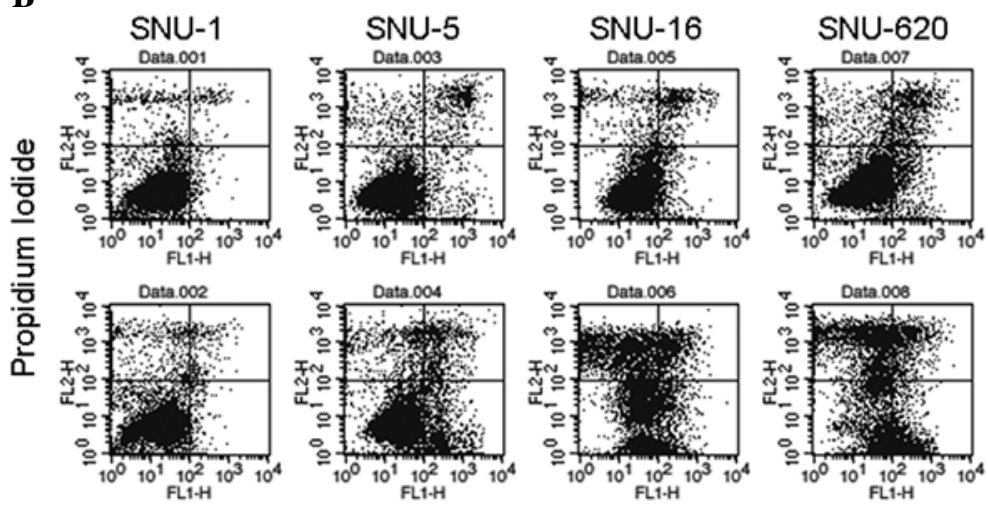

TRAIL $\mathrm{ng} / \mathrm{ml}$

0

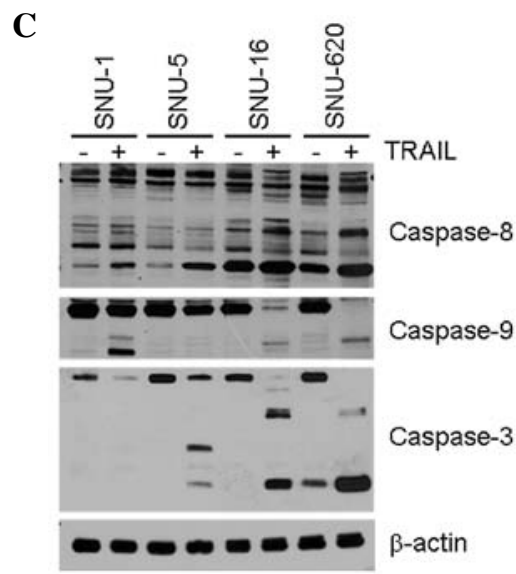

Figure 1. TRAIL-mediated apoptosis of the four gastric cancer cell lines. (A) Daily IC so $_{50}$ TRAIL-induced cell death of the four gastric cancer cells. The cells were plated one day prior to TRAIL treatment $(0,6.25,12.5,25,50$ or $100 \mathrm{ng} / \mathrm{ml})$, and MTT assay was carried out at 24,48 or $72 \mathrm{~h}$ after treatment. The percentage cell viability and $\mathrm{IC}_{50}$ were calculated as described in Materials and methods. The results shown are the average \pm standard deviation of three independent experiments. (B) Flow cytometric analysis of Annexin V binding. The cells were plated one day prior to TRAIL treatment (50 ng/ml), and $16 \mathrm{~h}$ after treatment, Annexin V binding/PI infiltration of the cells was measured by flow cytometry. The results shown are representatives of three independent experiments. (C) Activation of caspases upon TRAIL treatment. The activating fragmentation of caspase-3, -8 and -9 was detected by western blot analysis of cell lysates that were untreated or treated with $50 \mathrm{ng} / \mathrm{ml}$ TRAIL for $16 \mathrm{~h}$. Cell lysates were prepared and analyzed by western blot analysis, as described in Materials and methods. $\beta$-actin was used to monitor protein content on the western blots. The data shown are representatives of three independent experiments. 

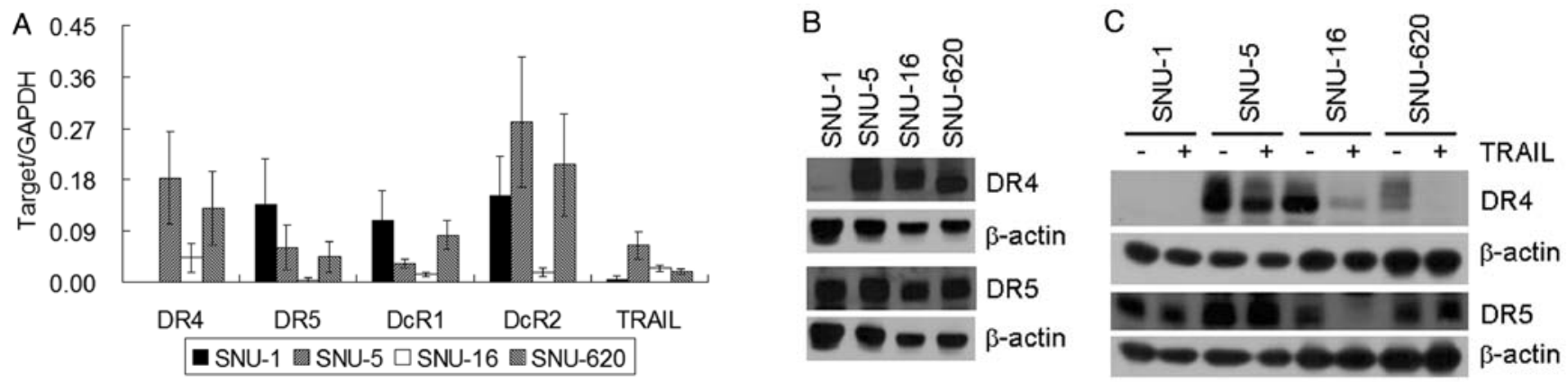

Figure 2. Expression of TRAIL receptors in the gastric cancer cells. (A) mRNA levels of TRAIL and TRAIL receptors. The mRNA expression of TRAIL and TRAIL receptors in the proliferating cells was examined by real-time RT-PCR. The target mRNA levels were normalized against those of GAPDH to monitor the total cDNA quantity in the PCR reaction. The data presented are the average \pm standard error $\left(x 10^{5}\right.$ for DR4, DR5 and TRAIL; x $10^{7}$ for DcR1; $10^{6}$ for DcR2) of seven independent experiments. (B and C) Western blot analysis of whole cell DR4 and DR5 proteins. Whole cell lysates were prepared from either proliferating cells (B) or TRAIL-treated cells $(50 \mathrm{ng} / \mathrm{ml})$ for $16 \mathrm{~h}(\mathrm{C})$. The expression of DR4 and DR5 was determined by western blot analysis as described in Materials and methods. $\beta$-actin was used to monitor protein content on the western blots. The data shown are representatives of three independent experiments.

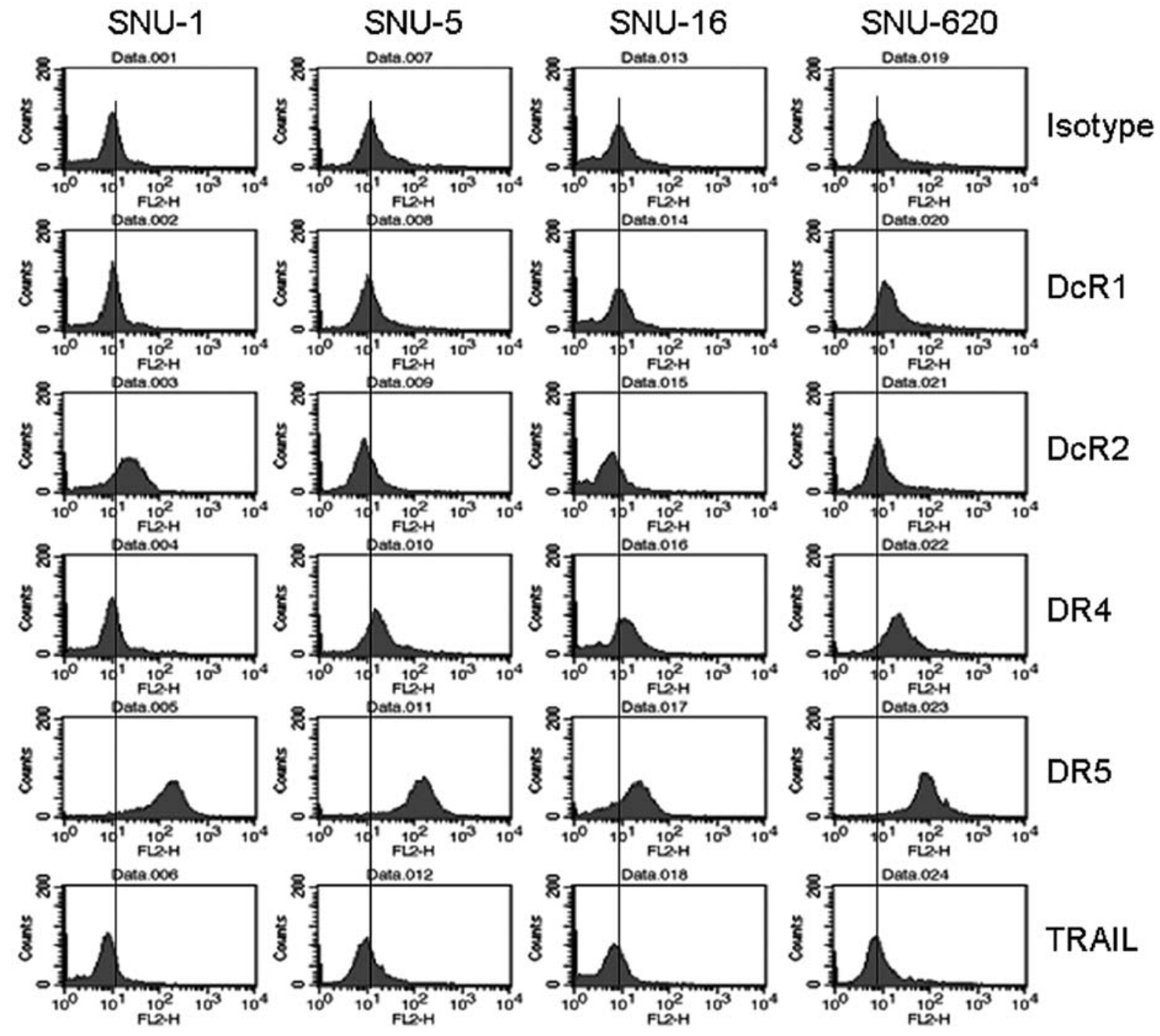

Figure 3. Cell surface expression of TRAIL receptors in the gastric cancer cells. The surface expression of TRAIL receptors was determined by flow cytometry after coupling cells with the indicated PE-conjugated antibodies. The non-specific binding of the antibodies was monitored using an antibody isotype control (vertical line). The data shown are representatives of three independent experiments.

was obvious in the SNU-5, SNU-16 and SNU-620 cells but not in the SNU-1 cells, which correlated with the mRNA results. In contrast to the mRNA levels, the expression of DcR1 on the cell surface was higher in the SNU-5 and SNU-620 cells than in the other cell lines, while surface DcR2 expression levels were noticeably higher in the SNU-1 cells. The expression of TRAIL 


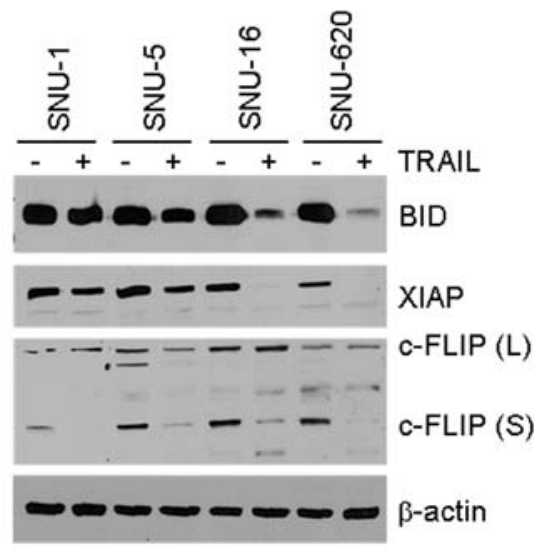

Figure 4. Expression of apoptosis modulators. The expression of the apoptosis modulators, Bid, XIAP and FLIP, was detected by western blot analysis of cell lysates that were untreated or treated with $50 \mathrm{ng} / \mathrm{ml}$ TRAIL for $16 \mathrm{~h}$. The cell lysates were prepared and analyzed by western blot analysis as described in Materials and methods. $\beta$-actin was used to monitor the protein content of the western blots. The data shown are representatives of three independent experiments.

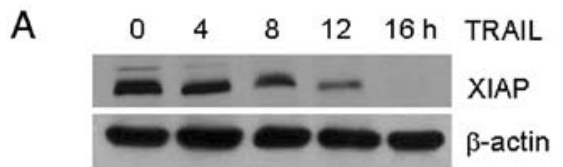

B

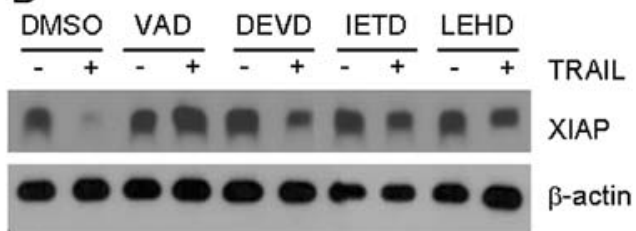

Figure 5. Fragmentation of XIAP in TRAIL-mediated cell death. (A) Decrease of XIAP protein expression in SNU-620 cells over time after TRAIL treatment. Western blot analysis of cell lysates prepared from cells treated with $50 \mathrm{ng} / \mathrm{ml} \mathrm{TRAIL}$ for the indicated time period was performed as described in Materials and methods. (B) Inhibition of XIAP cleavage by caspase inhibitors. SNU-620 cells were pre-treated with Z-VAD, DEVD, IETD or LETD $(20 \mu \mathrm{M}$ each) for $30 \mathrm{~min}$ and were then treated with $50 \mathrm{ng} / \mathrm{ml}$ TRAIL for $16 \mathrm{~h}$. The cell lysates were prepared and analyzed by western blot analysis as described in Materials and methods. $\beta$-actin was used to monitor the protein content on the blots. The data shown are representatives of three independent experiments.

was comparable among the four cell lines. Taken together, these results show that the expression of DR4 at the mRNA and protein levels and that of DcR2 on the cell surface positively correlated with the differential TRAIL sensitivity of the gastric cancer cells, whereas the expression of DR5 and DcR1 did not correlate with TRAIL sensitivity.

Expression of apoptosis modulators. Several pro- and antiapoptotic proteins, including the proteins of the $\mathrm{Bcl}-2$ and inhibitors of apoptosis (IAP) families, either stimulate or inhibit TRAIL-induced apoptosis (15-17). Therefore, to further understand the mechanism underlying the differential susceptibility to TRAIL-mediated apoptosis, we examined the expression of apoptosis modulators in the gastric cancer cell lines by western blot analysis. The fragmentation of Bid into tBid upon TRAIL treatment directly reflected the activation of caspase- 8 and TRAIL sensitivity (Fig. 4, upper panel). In addition, although the basal level of XIAP was similar in all four cell lines, XIAP was almost completely degraded in the TRAIL-sensitive SNU-16 and SNU-620 cells (Fig. 4, second panel from the top). However, the expression of the long and short forms of FLIP was relatively low in the SNU-1 cells and the short form completely disappeared upon TRAIL treatment in all cell lines (Fig. 4, third panel from the top). In conclusion, the fragmentation of Bid to tBid and XIAP degradation corresponded well to the TRAIL sensitivity of the gastric cancer cells, whereas the expression level of FLIP in the cells that were untreated or treated with TRAIL did not correlate with TRAIL sensitivity.

Since XIAP and cellular IAPs (cIAPs) are known to inhibit TRAIL-mediated apoptosis through interaction with caspases, XIAP cleavage may be required to promote TRAIL-mediated cell death (15). Otherwise, caspases activated by TRAIL cleave XIAP, thus facilitating apoptosis by a feed forward amplification loop (29). Therefore, we determined whether XIAP was cleaved by caspases activated by TRAIL treatment in the TRAILsensitive SNU-620 cells (Fig. 5). The amount of XIAP protein gradually decreased with time in the SNU-620 cells treated with TRAIL (Fig. 5A). However, the inhibition of caspase activity following pre-treatment with the pan-caspase inhibitor, Z-VAD, prevented the degradation of XIAP upon TRAIL treatment (Fig. 5B). These results suggest that XIAP may be cleaved by TRAIL-activated caspases, which accelerates the apoptosis induced by TRAIL.

\section{Discussion}

The differential susceptibility of cancer cells toward TRAILinduced apoptosis is an obstacle to the wide application of TRAIL as a cancer therapeutics. The effective application of TRAIL in cancer treatment necessitates the identification of potential indicators of TRAIL efficacy in cancer cells, prompting intense investigation into the molecular mechanisms of the TRAIL resistance of various cancer cells $(8,19)$. In this study, we aimed to identify potential indicators of TRAIL response through quantitative measurement of TRAIL cytotoxicity and perfomed a detailed analysis of the molecular mechanisms involved in the differential TRAIL sensitivity of four gastric cancer cell lines.

The efficacy of TRAIL decreased in the four gastric cancer cell lines in the following order: SNU-16 $\approx$ SNU-620 $>$ SNU-5 > SNU-1. This was further supported by an Annexin V binding assay and the caspase activation profile. TRAIL $\mathrm{IC}_{50}$ of the gastric cancer cells ranged between 23.8-87.1 ng/ml after $24 \mathrm{~h}$ of treatment, which was comparable to effective concentration of TRAIL (10-100 ng/ml) in the TRAIL-sensitive cancer cells. Of note, there was a positive correlation between TRAIL sensitivity and the location of the gastric cancer cell source. SNU-1 cells which were relatively resistant to TRAIL, were isolated from a solid tumor, while the other cell lines were established from ascites $(30,31)$. While the silencing of DR4 expression was implicated in the tempered TRAIL response of the SNU-1 cells in this study, no significant difference in the expression of DR4 was found between primary gastric carcinomas and metastasized ones from ascites (32). Thus, although tumor metastasis from ascites is different from ascites per se, the question of 
whether tumor cells in ascites are more sensitive to TRAIL than those in solid tumors remains to resolved.

The expression level of death-inducing and decoy TRAIL receptors has often been associated with TRAIL responsiveness (9). The downmodulation or loss of DR expression resulting from gene loss, mutations, epigenetic control and/ or post-translational regulation has been implicated in gastric cancer cells with TRAIL resistance (13,33-35). The expression of DR4 mRNA and protein was $\sim 1,000$-fold lower in the SNU-1 cells which were relatively resistant to TRAIL, than in the other tested cell lines, while the expression of DR5 and DcR1 was comparable between the cell lines. DR4 expression has been reported to correlate with TRAIL-induced apoptosis in various cancer cells despite the presence of functional DR5 (11,36). By contrast, DR5 has been shown to play a critical role in the TRAIL-mediated apoptosis of certain bladder cancer cells (37), suggesting that TRAIL preferentially exploits distinct DRs in different cells. In the gastric cancer cells, the silenced DR4 expression is the most critical component that determines TRAIL-mediated apoptosis. The downregulation of DR4 by promoter methylation has been reported in gastric carcinoma, which could abate the effectiveness of TRAIL $(33,34)$. However, since azacytidine, a DNA methylation inhibitor, did not restore DR4 expression (data not shown), its reduced expression in the SNU-1 cells was not attributed to downregulation by methylation.

Bid truncation into tBid by active caspase- 8 relays an extrinsic apoptotic signal triggered by TRAIL to the intrinsic apoptotic pathway (38). Akt activation renders ovarian cancer cells resistant to TRAIL by the downregulation of $\mathrm{Bid}$, suggesting that, in addition to the extrinsic pathway, the intrinsic pathway significantly contributes to TRAIL-induced apoptosis (39). While the expression level of Bid was similar among the gastric cancer cell lines, the full cleavage of Bid, as well as the appearance of active caspase- 9 and -3 fragments were obvious in the TRAIL-sensitive gastric cancer cells. In addition, although cleaved caspase-9 fragments were also observed in the SNU-1 cells, a significant amount of procaspase-9 still remained intact upon TRAIL treatment, including a significant amount of Bid. Thus, TRAIL sensitivity better correlates with the activation of caspases via the extrinsic pathway, which accentuates the importance of DRs and the subsequent activation of caspase- 8 in the gastric cancer cells.

The sensitivity of cancer cells to TRAIL-mediated cell death also correlates with intracellular levels of pro- and antiapoptotic proteins (9). Two forms of FLIP, a long form and a short form, inhibit TRAIL-mediated apoptosis by displacing caspase- 8 from the death-inducing signaling complex (DISC). The upregulation of FLIP by Akt has been suggested to be an inhibitory mechanism of TRAIL-induced apoptosis in SNU-216 gastric cancer cells (16). TRAIL response may also be influenced by the modulation of FLIP expression (40). However, the level of FLIP is not always indicative of the sensitivity to TRAIL (41). In the current study, there was no significant difference in the expression of both forms of FLIP among the gastric cancer cells, and, in all of the cell lines tested, the short form of FLIP disappeared upon TRAIL treatment (Fig. 4). Thus, the TRAIL susceptibility of the gastric cancer cells is likely independent of the FLIP expression and degradation level.
IAPs are inhibitors of apoptosis that interact with caspases and inhibit their activities (38). The expression of IAPs, particulary XIAP, has been reported to be inversely correlated with TRAIL sensitivity, and the modulation of XIAP expression influences TRAIL sensitivity in a number of cancer cell lines (15). Whereas a substantial amount of XIAP was detected in the gastric cancer cells, regardless of their susceptibility to TRAIL, XIAP expression diminished upon TRAIL treatment only in the TRAIL sensitive SNU-16 and SNU-620 cells. The inhibition of caspase activity by the caspase inhibitor, Z-VAD, fully rescued XIAP in the gastric cancer cells, suggesting that XIAP was cleaved by activated caspases in the TRAIL-treated gastric cancer cells (Fig. 5). XIAP degradation by activated caspases upon TRAIL treatment can further propagate apoptosis, which exerts positive feedback to enhance TRAILmediated apoptosis (29).

TRAIL is an emerging candidate for gastric cancer therapeutics and our results clearly support this possibility (23). However, the differential sensitivity to TRAIL-mediated apoptosis was also apparent among the gastric cancer cells tested. Our results demonstrated that the amounts of DR4 mRNA and protein coincided well with the TRAIL sensitivity of the gastric cancer cells. Therefore, the expression of DRs, particularly DR4, may serve as an indicator of TRAIL response in gastric cancer cells, although it would be too hasty to rule out other contributing factors. By contrast, since the expression of Bid, FLIP and XIAP was comparable between the gastric cancer cells, and since the cleavage of Bid to tBid and the degradation of XIAP were apparent only in the TRAIL-sensitive cells, these phenomena therefore play a limited role in enhancing TRAIL-induced apoptosis by a positive feedback loop in the gastric cancer cells.

\section{Acknowledgements}

The present study was supported by a grant from the National Research Foundation of Korea (no. 2010-0013381 to D.K.).

\section{References}

1. Wiley SR, Schooley K, Smolak PJ, et al: Identification and characterization of a new member of the TNF family that induces apoptosis. Immunity 3: 673-682, 1995.

2. Bouralexis S, Findlay DM and Evdokiou A: Death to the bad guys: targeting cancer via Apo2L/TRAIL. Apoptosis 10: 35-51, 2005.

3. Simonet WS, Lacey DL, Dunstan CR, et al: Osteoprotegerin: a novel secreted protein involved in the regulation of bone density. Cell 89: 309-319, 1997.

4. Almasan A and Ashkenazi A: Apo2L/TRAIL: apoptosis signaling, biology, and potential for cancer therapy. Cytokine Growth Factor Rev 14: 337-348, 2003.

5. Zauli G and Secchiero P: The role of the TRAIL/TRAIL receptors system in hematopoiesis and endothelial cell biology. Cytokine Growth Factor Rev 17: 245-257, 2006.

6. Ashkenazi A, Pai RC, Fong S, et al: Safety and antitumor activity of recombinant soluble Apo2 ligand. J Clin Invest 104: 155-162, 1999.

7. Xiang H, nguyen CB, Kelley SK, Dybdal N and Escandon E: Tissue distribution, stability, and pharmacokinetics of Apo2 ligand/tumor necrosis factor-related apoptosis-inducing ligand in human colon carcinoma COLO205 tumor-bearing nude mice. Drug Metab Dispos 32: 1230-1238, 2004.

8. Johnstone RW, Frew AJ and Smyth MJ: The TRAIL apoptotic pathway in cancer onset, progression and therapy. Nat Rev Cancer 8: 782-798, 2008. 
9. Zhang L and Fang B: Mechanisms of resistance to TRAILinduced apoptosis in cancer. Cancer Gene Ther 12: 228-237, 2005.

10. Hopkins-Donaldson S, Ziegler A, Kurtz S, et al: Silencing of death receptor and caspase- 8 expression in small cell lung carcinoma cell lines and tumors by DNA methylation. Cell Death Differ 10: 356-364, 2003.

11. Kim K, Fisher MJ, Xu SQ and el-Deiry WS: Molecular determinants of response to TRAIL in killing of normal and cancer cells. Clin Cancer Res 6: 335-346, 2000.

12. Ozoren N, Fisher MJ, Kim K, et al: Homozygous deletion of the death receptor DR4 gene in a nasopharyngeal cancer cell line is associated with TRAIL resistance. Int J Oncol 16: 917-925, 2000.

13. Wajant H, Pfizenmaier K and Scheurich P: TNF-related apoptosis inducing ligand (TRAIL) and its receptors in tumor surveillance and cancer therapy. Apoptosis 7: 449-459, 2002.

14. Sanlioglu AD, Dirice E, Aydin C, Erin N, Koksoy S and Sanlioglu S: Surface TRAIL decoy receptor-4 expression is correlated with TRAIL resistance in MCF7 breast cancer cells. BMC Cancer 5: 54, 2005.

15. Cummins JM, Kohli M, Rago C, Kinzler KW, Vogelstein B and Bunz F: X-linked inhibitor of apoptosis protein (XIAP) is a nonredundant modulator of tumor necrosis factor-related apoptosis-inducing ligand (TRAIL)-mediated apoptosis in human cancer cells. Cancer Res 64: 3006-3008, 2004.

16. Nam SY, Jung GA, Hur GC, et al: Upregulation of FLIP(S) by Akt, a possible inhibition mechanism of TRAIL-induced apoptosis in human gastric cancers. Cancer Sci 94: 1066-1073, 2003.

17. Sun SY, Yue P, Zhou JY, et al: Overexpression of Bcl2 blocks TNF-related apoptosis-inducing ligand (TRAIL)-induced apoptosis in human lung cancer cells. Biochem Biophys Res Commun 280: 788-797, 2001

18. Chen JJ, Knudsen S, Mazin W, Dahlgaard J and Zhang B: A 71-gene signature of TRAIL sensitivity in cancer cells. Mol Cancer Ther 11: 34-44, 2012.

19. Mahalingam D, Szegezdi E, Keane M, de Jong S and Samali A TRAIL receptor signalling and modulation: are we on the right TRAIL? Cancer Treat Rev 35: 280-288, 2009.

20. Ferlay J, Shin HR, Bray F, Forman D, Mathers C and Parkin DM: Estimates of worldwide burden of cancer in 2008: GLOBOCAN 2008. Int J Cancer 127: 2893-2917, 2010.

21. Cervantes A, Rosello S, Roda D and Rodriguez-Braun E: The treatment of advanced gastric cancer: current strategies and future perspectives. Ann Oncol 19 (Suppl 5): v103-v107, 2008.

22. Yoong J, Michael M and Leong T: Targeted therapies for gastric cancer: current status. Drugs 71: 1367-1384, 2011.

23. Qiao L and Wong BC: Targeting apoptosis as an approach for gastrointestinal cancer therapy. Drug Resist Updat 12: 55-64, 2009.

24. Belkhiri A, Zhu S, Chen Z, Soutto M and El-Rifai W: Resistance to TRAIL Is Mediated by DARPP-32 in Gastric Cancer. Clin Cancer Res 18: 3889-3900, 2012.

25. Qu J, Zhao M, Teng Y, et al: Interferon-alpha sensitizes human gastric cancer cells to TRAIL-induced apoptosis via activation of the c-CBL-dependent MAPK/ERK pathway. Cancer Biol Ther 12: 494-502, 2011

26. Liu J, Qu XJ, Xu L, et al: Bortezomib synergizes TRAIL-induced apoptosis in gastric cancer cells. Dig Dis Sci 55: 3361-3368, 2010.
27. Huynh KM, Soh JW, Dash R, Sarkar D, Fisher PB and Kang D: FOXM1 expression mediates growth suppression during terminal differentiation of HO-1 human metastatic melanoma cells. J Cell Physiol 226: 194-204, 2011

28. Uno K, Inukai T, Kayagaki N, et al: TNF-related apoptosisinducing ligand (TRAIL) frequently induces apoptosis in Philadelphia chromosome-positive leukemia cells. Blood 101: 3658-3667, 2003

29. Hornle M, Peters N, Thayaparasingham B, Vorsmann H, Kashkar H and Kulms D: Caspase-3 cleaves XIAP in a positive feedback loop to sensitize melanoma cells to TRAIL-induced apoptosis. Oncogene 30: 575-587, 2011.

30. Park JG, Frucht H, LaRocca RV, et al: Characteristics of cell lines established from human gastric carcinoma. Cancer Res 50: 2773-2780, 1990

31. Park JG, Yang HK, Kim WH, et al: Establishment and characterization of human gastric carcinoma cell lines. Int J Cancer 70: 443-449, 1997.

32. Koyama S, Koike N and Adachi S: Expression of TNF-related apoptosis-inducing ligand (TRAIL) and its receptors in gastric carcinoma and tumor-infiltrating lymphocytes: a possible mechanism of immune evasion of the tumor. J Cancer Res Clin Oncol 128: 73-79, 2002

33. Lee KH, Lim SW, Kim HG, et al: Lack of death receptor 4 (DR4) expression through gene promoter methylation in gastric carcinoma. Langenbecks Arch Surg 394: 661-670, 2009.

34. Park WS, Lee JH, Shin MS, et al: Inactivating mutations of KILLER/DR5 gene in gastric cancers. Gastroenterology 121: 1219-1225, 2001

35. Fisher MJ, Virmani AK, Wu L, et al: Nucleotide substitution in the ectodomain of trail receptor DR4 is associated with lung cancer and head and neck cancer. Clin Cancer Res 7: 1688-1697, 2001.

36. Lemke J, Noack A, Adam D, et al: TRAIL signaling is mediated by DR4 in pancreatic tumor cells despite the expression of functional DR5. J Mol Med 88: 729-740, 2010.

37. Szliszka E, Mazur B, Zydowicz G, Czuba ZP and Krol W: TRAIL-induced apoptosis and expression of death receptor TRAIL-R1 and TRAIL-R2 in bladder cancer cells. Folia Histochem Cytobiol 47: 579-585, 2009.

38. Aggarwal BB, Bhardwaj U and Takada Y: Regulation of TRAILinduced apoptosis by ectopic expression of antiapoptotic factors. Vitam Horm 67: 453-483, 2004.

39. Goncharenko-Khaider N, Lane D, Matte I, Rancourt C and Piche A: The inhibition of Bid expression by Akt leads to resistance to TRAIL-induced apoptosis in ovarian cancer cells. Oncogene 29: 5523-5536, 2010.

40. Seal S, Hockenbery DM, Spaulding EY, Kiem HP, Abbassi N and Deeg HJ: Differential responses of FLIPLong and FLIPShortoverexpressing human myeloid leukemia cells to TNF-alpha and TRAIL-initiated apoptotic signals. Exp Hematol 36: 1660-1672, 2008.

41. Zhang XD, Franco A, Myers K, Gray C, Nguyen T and Hersey P. Relation of TNF-related apoptosis-inducing ligand (TRAIL) receptor and FLICE-inhibitory protein expression to TRAILinduced apoptosis of melanoma. Cancer Res 59: 2747-2753, 1999. 